\title{
Une entreprise en territoire occupé : Arbel à Douai
} (1914-1919)

Anne Callite

\section{CpenEdition}

\section{Journals}

Édition électronique

URL : https://journals.openedition.org/rhcf/511

DOI : 10.4000/rhcf.511

\section{Éditeur}

Rails \& histoire

\section{Édition imprimée}

Date de publication : 1 novembre 2006

ISSN : 0996-9403

\section{Référence électronique}

Anne Callite, « Une entreprise en territoire occupé : Arbel à Douai (1914-1919)», Revue d'histoire des chemins de fer [En ligne], 35 | 2006, mis en ligne le 05 avril 2011, consulté le 22 avril 2022. URL : http:// journals.openedition.org/rhcf/511 ; DOI : https://doi.org/10.4000/rhcf.511 


\section{Anne CALLITE}

Docteur en histoire, université Charles-de-Gaulle - Lille 3

\section{Une entreprise en territoire occupé : Arbel à Douai (1914-1919)}

Le groupe des usines de locomotives et de wagons dans ce qu'on appellera bientôt le «territoire occupé » ${ }^{1}$ comprend en 1916 seize établissements, dont quinze pour la région Nord - Pas-de-Calais, ayant un capital de 130 millions de francs et occupant 27817 ouvriers. Leur part dans la production française de matériel ferroviaire est estimée à $41 \%^{2}$. Dans ce chiffre sont compris les ateliers de fabrication de signaux pour chemins de fer, qui sont toutefois peu importants. Presque tous ces établissements sont de grandes entreprises, mais une importante diversité règne : certains sont présents dans l'ensemble des fabrications destinées au chemin de fer, d'autres sont spécialisés dans un produit. Tous cependant se livrent à une diversification quasi obligatoire : matériel de sucrerie, charpentes, ponts, etc. Les Ateliers de construction du Nord de la France (ANF, à Crespin-Blanc-Misseron) présentent quant à eux la particularité d'avoir spécialisé deux de leurs filiales, pour le wagonnage et pour les grosses locomotives. Rappelons, par ailleurs, que sur les six constructeurs qui forment en France l'industrie de la locomotive avant 1914, trois d'entre eux sont nordistes ${ }^{3}$ : la Société française de constructions mécaniques - anciens Établissements Cail (SFCM, à Denain), la Compagnie de Fives-Lille (Lille) et la Société franco-belge de matériel de chemin de fer (Raismes).

1- Les territoires occupés comprenaient tout ou partie de dix départements : les Ardennes, l'Aisne, le Nord, la Meuse, la Meurthe-et-Moselle, le Pas-de-Calais, la Somme, la Marne, l'Oise, les Vosges. Seules les Ardennes sont occupées en totalité. Le Nord et le Pas-de-Calais sont occupés dans une proportion de respectivement 70 et $25 \%$ de leur territoire.

2- L'Industrie en France occupée, ouvrage établi par le Grand quartier général allemand en 1916, Paris, Imprimerie nationale, 1923, 534 p., p. 490.

3- Ces entreprises forment avant la guerre un cartel, dénommé « groupement des constructeurs de locomotives ", avec trois autres firmes: Schneider et Cie, la Société des Batignolles et la Société alsacienne de constructions mécaniques. 
La capacité de production des établissements de matériel ferroviaire installés dans le seul département du Nord est au total d'environ 500 locomotives et 9000 wagons par an, soit approximativement 95 et $70 \%$ des besoins moyens de la France. Quatre grands constructeurs de matériel ferroviaire sont présents dans le Valenciennois. En tête vient la Société française de constructions mécaniques, qui représente à elle seule 22 à $27 \%$ de la production française de locomotives : en 1907, la SFCM a réussi à porter sa production annuelle de locomotives à une centaine de machines. Fin 1911, elle estime que celle-ci dépassera bientôt les 150 locomotives (environ 10000 tonnes), ce qui représenterait alors $26,5 \%$ de la production totale française. Il faut préciser par ailleurs que, au cours des dix années précédant la guerre, toutes les usines du matériel roulant en France ont considérablement accru leur capacité de production qui, partant en 1904 de 250 à 300 locomotives et 8000 voitures et wagons, est parvenue aux chiffres suivants : 600 à 700 locomotives (suivant le poids), 9000 voitures et 18000 wagons ${ }^{4}$.

Si l'on compare la moyenne des besoins annuels des chemins de fer français avec la capacité de production des ateliers de locomotives et de wagons, il est clair que ces établissements sont en état de satisfaire les besoins à la condition de répartir convenablement les commandes. Or, tant les prix que la planification des besoins par les compagnies de chemins de fer s'y opposent. De 1910 à 1913, 18,5 \% des commandes de locomotives passées par les réseaux français le sont auprès de constructeurs étrangers. Des observateurs allemands peuvent alors écrire en 1916 : « l'irrégularité du travail est très préjudiciable, surtout aux fabriques de locomotives et nuit aux résultats 5 .»

Les autres constructeurs ont une capacité moindre en matière de matériel de traction, mais leur production ferroviaire inclut voitures et wagons, même si la Compagnie de Fives-Lille ne réalise pas de voitures. Seule la Franco-belge construit tous les types de matériel roulant, ce qui fait d'elle à cette époque le constructeur ferroviaire le plus complet du secteur. Elle fournit alors en moyenne par an 50 à 60 locomotives, 1500 à 2000 voitures et wagons, tandis que la Compagnie générale de construction (à Marly-lez-Valenciennes), avec ses 3000 wagons annuels, présente une capacité de production moitié moindre de celle de la Franco-belge. Si nous comparons la Franco-belge avec les ANF, nous constatons que l'équipement des deux entreprises est très différent alors

4- Chambre syndicale des fabricants et constructeurs. Assemblée générale ordinaire. 17 juin 1918. Fonctionnement de la Chambre syndicale pendant l'année 1917-1918.

5- L'Industrie en France occupée, op. cit., p. 62. 
que leurs productions sont relativement similaires : locomotives, voitures, wagons... Les ANF sont particulièrement bien équipés pour le forgeage : l'inventaire des machines restant en 1919 dans l'usine de BlancMisseron et détériorées par les Allemands signale l'existence de 17 marteaux-pilons dont 14 ont une masse tombante inférieure à $500 \mathrm{~kg}$. Huit d'entre eux ont été installés dès l'exercice 1898-1899 et une forge avec marteaux-pilons pour le matriçage des roues de chemins de fer a été montée dès l'exercice suivant (tabl. 1$)^{6}$.

Tableau 1. Firmes et moyens d'exploitation des entreprises de construction de matériel roulant ferroviaire en $1916^{7}$

\begin{tabular}{|c|c|c|r|}
\hline \multicolumn{2}{|c|}{ Nom bre } & \multicolumn{2}{r}{ Ouvrie } \\
\hline $\begin{array}{c}\text { Firmes (dont usines en } \\
\text { cours d'agrandissement) }\end{array}$ & $\begin{array}{c}\text { Exploitations } \\
\text { annexes }\end{array}$ & Hommes & Étrangı \\
\hline $15(3)$ & 3 & 21590 & 3631 \\
\hline \multicolumn{4}{|c|}{ Moyens d'exploitation } \\
\hline \multicolumn{4}{|c|}{ Machines m otrice } \\
\hline $\begin{array}{c}\text { Chaudières à vapeur } \\
\text { Total (dont utilisables) }\end{array}$ & $\begin{array}{c}|c| \\
\text { Électriques } \\
\text { Total (dont } \\
\text { utilisables) }\end{array}$ & $\begin{array}{c}\text { À vapeur (dont } \\
\text { utilisables) }\end{array}$ & $\begin{array}{r}\text { À combl } \\
\text { utilisab }\end{array}$ \\
\hline 71 (67) & 343 (288) & 23 (21) & $3(2 ;$ \\
\hline
\end{tabular}

Dans ces entreprises de constructions mécaniques, la gestion est incontestablement dynamique et tend à le devenir de plus en plus : aussi Odette Hardy-Hémery nous annonce-t-elle un taux annuel de croissance des investissements de 25,24\% de 1904 à $1913^{8}$, tandis que, au niveau de l'emploi, la branche ferroviaire triple au moins ses effectifs entre 1900 et 1914. Bien sûr, ce dynamisme présente des degrés divers.

6- Archives départementales du Nord (désormais AD Nord) 10 R 4297. Dossier de dommages de guerre. Société Franco-Belge. AD Nord 10 R 5492. Dossier de dommages de guerre. Ateliers de construction du nord de la France.

7- L'Industrie en France occupée, op. cit., p. 240 et 241.

8- Odette HARDY-HEMERY, De la croissance à la désindustrialisation. Un siècle dans le Valenciennois, Paris, Presses de la Fondation nationale des sciences politiques, 1984, 401 p., p. 84. 
Une enquête réalisée par le Grand quartier général allemand en 1916 révèle ainsi deux lacunes assez surprenantes : «si l'on excepte un petit nombre d'établissements de constructions mécaniques d'une importance prépondérante, il est vrai, les exploitations de cette région sont une déception pour l'homme du métier. Si on les compare à l'industrie des machines, souvent très importante et travaillant d'après des méthodes modernes, du département de la Seine par exemple, on ne peut se défendre que malgré les conditions favorables où elles se trouvent naturellement, ou peut-être à cause de ces conditions mêmes leur situation est très négligée. La tendance propre aux entrepreneurs français d'utiliser les installations existantes tant qu'elles ne sont pas une cause de pertes, peut être constatée nettement dans la région occupée 9 . » Il nous faut démentir ces allégations : à la veille de la guerre, la plupart des usines sont neuves.

\section{Arbel, une entreprise florissante}

Lucien $\mathrm{Arbel}^{10}$, à l'origine de la société homonyme, est entré dans l'industrie en 1856 et exploite à Rive-de-Gier, en association avec les frères Deflaissieux ${ }^{11}$, le brevet que ces derniers ont déposé en 1854 pour l'invention et la fabrication de la roue en fer forgé par matriçage au marteau-pilon. En 1869, L. Arbel fonde les Forges de Couzon, situées sur la rivière de Couzon, dans un faubourg de Rive-de-Gier dans le département de la Loire, sur un terrain d'une superficie de 5 hectares. Ces usines, qui se spécialisent ensuite dans la fabrication des roues en fer forgé et des essieux montés de chemins de fer, prennent bientôt un développement important. Outre les pièces destinées aux chemins de fer et tramways, les forges réalisent des pièces de grosse et petite forge, des obus et éléments de canon, des outils pneumatiques et des pièces mécaniques ${ }^{12}$.

9- L'Industrie en France occupée, op. cit., p. 58.

10- Lucien Arbel est ingénieur des Arts et Métiers. Il deviendra par la suite député puis sénateur de la Loire (La Passerelle, $\mathrm{n}^{\circ} 11$, septembre 1957). Voir également L'Illustration, $\mathrm{n}^{\circ} 1030$ (22 novembre 1862), p. 347-348.

11- Les Capitalistes en France: 1780-1914, présenté par Louis BERGERON, Paris, Gallimard, 1978, 233 p., p. 59-60.

12- Archives nationales (Centre des archives du monde du travail, désormais CAMT) 70 AQ 90. Forges de Douai. Apport des Forges de Couzon aux Établissements Arbel. 18 janvier 1908 . 
En 1890, Pierre Arbel ${ }^{13}$, fils de Lucien, préoccupé par la crise que traverse alors l'industrie de la Loire, entreprend une série de voyages pour étudier les conditions commerciales et industrielles des régions du Nord et de l'Est. À la suite de cette enquête, les Établissements Arbel s'entendent avec la ville de Douai pour acheter, sur les anciens remparts déclassés, un lot de 5 hectares de terrain, mitoyen de la gare de Douai. La ville de Douai réserve en effet 110 hectares de terrains aux entreprises : «De 1892 à 1914, 179 parcelles sont vendues au prix minimum d'un demi-franc le mètre carré hors remparts, et de la un franc à l'intérieur, la plupart avant $1900^{14}$. » De plus, la ville s'engage à rembourser pendant deux ans les droits d'octroi sur les matières premières employées pour l'installation d'usines ${ }^{15}$. Elle aide ainsi au développement de sociétés déjà établies, telles les usines Cail qui s'agrandissent en 1892, 1896 et 1900 et incite à l'installation de nouvelles firmes : on voit bientôt s'édifier les Usines Bréguet (aéronautique), des brasseries, des tanneries, et Arbel. La Société anonyme des Forges de Douai est fondée en avril 1894 avec un capital de 1200000 francs $^{16}$. Pierre Arbel est nommé administrateurdélégué et Lucien Arbel devient administrateur. La nouvelle société a pour objet « l'industrie et le commerce des forges et de la métallurgie [...] ; l'acquisition et la vente de tous brevets d'invention et procédés de fabrication relatifs à la dite industrie de toute clientèle, achalandage et marchandises $»^{17}$. Mais peu après août 1896 , et bien que primitivement établie pour la fabrication des pièces de grosse et moyenne forge, les Forges de Douai entreprennent l'emboutissage des tôles, prenant par la suite un développement intéressant comme procédé de construction. Car, avant d'être un constructeur ferroviaire, Arbel est une société spécialisée dans l'emboutissage.

13- Pierre Arbel est l'un des commissaires rapporteurs de l'Exposition internationale de Chicago (Pierre ARBEL, Mines. Exploitation des mines et Métallurgie, 1894 [Exposition de Chicago en 1893. Rapports. Comité 12]). Voir aussi la maîtrise de Laure HENNEQUIN, « La société Arbel de 1894 à 1939 : un demi-siècle d'histoire technique, économique et sociale de la vie d'une entreprise française de construction mécanique », Lille 3 , 1993.

14- Michel ROUCHE (dir.), Histoire de Douai, 1985, p. 214 et 235-236.

15- ANC (CAMT) 70 AQ 1. Forges de Douai. Conseil d'administration. 15 mai 1895.

16- Ibid, 24 avril 1894.

17- ANC (CAMT) 70 AQ 90. Établissements Arbel. Actes constitutifs de la Société des Forges de Douai (31 janvier 1894). 
En 1900, un nouvel atelier est installé, abritant un four Martin de 7 tonnes et une fabrication de roues en acier forgé laminé. La forge, l'ajustage et l'emboutissage sont considérablement développés et on procède, notamment, à l'installation d'une presse à forger de 3000 tonnes. L'usine $\mathrm{n}^{\circ} 1$ compte alors trois halls parallèles correspondant aux trois activités de la firme.

\section{La fabrication des wagons de grande capacité}

Continuant leur développement, les Forges de Douai acquièrent en 1901, de la Leeds Forge ${ }^{18}$, l'exclusivité de ses procédés de fabrication « Fox » relatifs aux pièces embouties entrant dans la construction du matériel de chemin de fer. Elles réalisent, dès 1903, une première série de wagons de grande capacité destinée à la Compagnie du Midi, bientôt suivie de commandes importantes pour la Compagnie du Nord. Pierre Arbel dépose également un brevet de châssis emboutis pour l'automobile. Cette extension d'activité oblige les Établissements Arbel, qui à l'époque s'appellent encore Forges de Douai, à acquérir en 1908 un terrain de 3 hectares situé à l'intérieur de la ville sur lequel est bâti un atelier que l'on appelle usine $n^{\circ} 2$. Cette usine se consacre à la construction et à la réparation de matériel roulant. Dans le même temps, les Forges de Douai développent les fabrications spéciales de l'artillerie et des constructions navales : elles réalisent des éléments de canons et le premier mortier de 370 fait avant la guerre ${ }^{19}$. Elles montent également la fabrication des arbres forés pour cuirassés et la mobilisation les surprend achevant la fabrication des lignes d'arbres des cuirassés du programme 1913.

L'activité de «l'Usine Arbel» est alors intense. En janvier 1908 apparait pour la première fois la nouvelle raison sociale «Établissements Arbel, Forges de Douai, Forges de Couzon ", qui consacre la fusion des deux sociétés. La même année, la société Arbel devient propriétaire du champ de manœuvres de la garnison de Douai représentant 41 hectares de terrains situés sur la "Berce Gayant», de l'autre côté de la voie ferrée. En 1910, Pierre Arbel établit le programme d'une grande usine métallurgique appelée usine $n^{\circ} 3$ et comprenant, en 1914, une aciérie Martin, un laminoir à grosses tôles, deux laminoirs à tôles fines, et un atelier de trains montés avec forge à essieux, laminoirs à roues et

18- Nos usines métallurgiques dévastées (1914-1918). Monographies de quelques grandes usines métallurgiques françaises détruites par les Allemands, Paris, Éditions de la Revue de métallurgie, 1921, 234 p., p. 70.

19- La Passerelle, $\mathrm{n}^{\circ} 11$ (septembre 1957). 
laminoirs à bandages. En 1913-1914, l'atelier d'emboutissage de la firme Arbel, que celle-ci qualifie elle-même d' $"$ unique en Europe $»^{20}$, comporte 14 presses à emboutir, produit annuellement 10000 châssis d'automobiles et 10000 tonnes d'emboutis de toute nature (tabl. 2).

Tableau 2. Ateliers de Douai de la Société Arbel en 1914²1

\begin{tabular}{|l|}
\hline \multicolumn{1}{|c|}{ Puissance de production } \\
\hline Fours Martin (capacité de production) \\
\hline Cubilots (capacité) \\
\hline Laminoir grosses tôles \\
\hline Laminoir tôles fines \\
\hline Laminoir roues de chemin de fer \\
\hline Laminoir bandages de chemin de fer \\
\hline Essieux \\
\hline Pièces de forge, artillerie, constructions navales, divers \\
\hline Presse à fer de $3000 \mathrm{t}$ \\
\hline Presses à forger de $1500-1000$-600 $\mathrm{t}$ \\
\hline Pilons dont 1 de $30 \mathrm{t}$ (capacité totale) \\
\hline
\end{tabular}

En l'espace de 18 ans, la société Arbel, qui a débuté sur un terrain de cinq hectares avec 40 ouvriers, s'est développée à tel point qu'en 1914 elle occupe une surface de 55 hectares et emploie 2500 ouvriers. Les trois usines d'Arbel forment à Douai un ensemble de surfaces bâties de $86650 \mathrm{~m}^{2}$.

\section{Un afflux de commandes synonyme d'engorgement industriel}

Cette vitalité est cependant précaire. Le dynamisme des constructeurs mécaniques masque mal les difficultés inhérentes à la construction du matériel de chemins de fer, les légendaires commandes aléatoires et les retards à la livraison. En effet, l'importance des commandes passées quelques années avant la guerre prend de court

20- AD Nord 10 R 4563. Dossier de dommages de guerre. Société Arbel. Note du 16 décembre 1918. Nos usines métallurgiques dévastées (1914-1918)..., op. cit., p. 73 : le chiffre de 16000 emboutis est ici annoncé.

21- AD Nord 10 R 4563. Dossier de dommages de guerre. Société Arbel. Note du 16 décembre 1918. 
l'ensemble de l'industrie ferroviaire française, au point que la Compagnie du Nord considère les retards comme "normaux », sans pour autant oublier d'agiter « devant leurs yeux avec insistance le spectre des pénalités $»^{22}$. Ainsi, si nous prenons en exemple une commande du Nord auprès de la société Arbel de 100 wagons SSy livrables à la fin mars 1912, seuls 20 pouvaient être livrés à cette date, le reste de la commande s'étalant jusqu'en juillet 1912. Ce n'est pas un problème de capacité de production car, en 1913-1914, l'atelier de wagonnage et de trains montés de Douai produit 240 tenders, 3000 wagons de grande capacité, 12000 essieux, 45000 roues et 45000 bandages $^{23}$.

Mais, face à l'afflux des commandes de chemins de fer, les ateliers sont engorgés. La situation est identique aux $\mathrm{ANF}^{24}$, chez Carel et Fouché, à la CGC, etc. Parallèlement à cette forte activité des constructeurs ferroviaires, le trafic du réseau du Nord est intense, ce qui met à nouveau en évidence la relation entre l'activité des réseaux et celle des constructeurs. À l'automne 1913, le trafic journalier atteint 27000 wagons chargés ${ }^{25}$.

\section{Les préparatifs de guerre}

Cette réussite a cependant favorisé des frictions avec les firmes allemandes. Aussi, le grand quartier qénéral allemand annonce-t-il en 1916 que le constructeur Arbel est techniquement supérieur aux usines allemandes : "pour la fabrication de grandes tôles pressées, il peut presser des cadres de tôles allant jusqu'à 24 mètres de long. C'est pourquoi il avait, avant la guerre, enlevé à ses concurrents allemands,

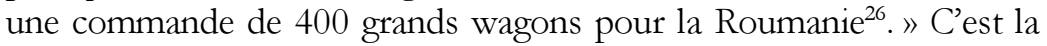
même chose pour les essieux, les roues... Arbel est une usine modèle.

22- ANC (CAMT) 202 AQ 1267. Compagnie du Nord. Livraison du matériel par les constructeurs. Situation et états mensuels des commandes du matériel roulant en cours d'exécution (1867-1930). Dossier matériel. Année 1912. Lettre de Asselin, ingénieur en chef du matériel à Javary, ingénieur en chef au service de l'exploitation, 6 mars 1912.

23- Nos usines métallurgiques dévastées (1914-1918)..., op. cit., p. 73.

24- ANC (CAMT) 202 AQ 1267. Compagnie du Nord. Livraison du matériel par les constructeurs. Situation et états mensuels des commandes du matériel roulant en cours d'exécution (1867-1930). Relevé au $1^{\text {er }}$ juin 1912.

25- Pierre DAUZET, Le Siècle des chemins de fer en France (1821-1938), Fontenay-auxRoses, impr. de Bellenand, 1948, 379 p., p. 227.

26- L'Industrie en France occupée, op. cit., p. 53. 
Le même constat est établi pour la SFCM et la Compagnie de FivesLille : « la visite des établissements Cail à Denain et de ceux de Fives-Lille a eu pour résultat de faire découvrir que ces établissements avaient entrepris un effort énergique pour faire obstacle à l'importation de machines allemandes en améliorant leurs propres produits ${ }^{27}$. » La guerre va bientôt, à sa manière, résoudre et annihiler tous les problèmes de concurrence et de jalousie. D'usines, il ne sera bientôt plus question.

Dès 1911, les industriels sont conscients des risques de conflit sur le continent européen. Cette année-là, Arbel, arguant de l'importance de ses usines pour les fabrications militaires, tente d'obtenir une « mise en sursis d'appel » pour son personnel, ou tout simplement le classement en « non affectation », mais la demande n'aboutit pas, le ministère de la Guerre refusant de reconnaittre un caractère d'absolue nécessité aux fabrications d'Arbel ${ }^{28}$. Les événements s'accélèrent très vite en juillet 1914. Le 29 juillet, le directeur général d'Arbel, Brochard, convoque d'urgence le conseil d'administration et lui annonce qu'il a loué un coffre-fort près de la Société générale pour y sauvegarder les fonds disponibles, les livres de comptabilité, les contrats, les registres de procèsverbaux... Mais la guerre devant être courte, le coffre n'est loué que pour trois mois!

Très vite, la mobilisation fait chuter les effectifs et désorganise les usines en les privant brusquement de la plupart de leurs administrateurs, ingénieurs, employés et ouvriers. Au siège d'Arbel, il reste 21 hommes sur 52, 305 sur 505 à Couzon, 487 sur 1010 à Douai - usine 1 et 212 sur 403 à Douai - usine 3. «Le dimanche 2 août 1914, jour de la déclaration de guerre, fut marqué par le départ des trois quarts du personnel ouvrier et employé des Forges de Douai ; chacun faisant son devoir répondait à l'ordre de mobilisation qu'il avait reçu. Toutes les dispositions avaient, du reste, été prises, par nos services, pour faciliter

27-Ibid., p. 61.

28- ANC (CAMT) 70 AQ 5. Établissements Arbel. Conseil d'administration. 7 décembre 1911.

29- AD Nord 10 R 4565. Dossier de dommages de guerre. Établissements Arbel à Douai. Rapport sur les événements survenus aux Établissements Arbel - Usines de Douai pendant la guerre de 1914-1918, dressé par M. Godon, s/agent administratif des Forges de Douai. 22 avril 1919.

30- ANC (CAMT) 70 AQ 5. Établissements Arbel. Conseil d'administration. 29 juillet et 28 novembre 1914 . 
l'accomplissement de cet élan patriotique ${ }^{29}$. » Mais les administrateurs d'Arbel sont surtout admiratifs du « beau geste » des fils de Pierre Arbel, Antoine et Luc, qui se sont engagés dès le début des hostilités ${ }^{30}$.

\section{Un champ de bataille immense}

Dans un premier temps, les usines s'appliquent à remanier les divers services et ateliers pour les équilibrer, dans la mesure du possible, avec les hommes non appelés sous les drapeaux. Ce premier effort devait être vain. Dès la déclaration de guerre ${ }^{31}$, certaines usines ont arrêté leur production (Escaut-et-Meuse à Anzin), puis au fil des événements, les entreprises cessent leurs activités : le 25 août 1914, jour de l'arrivée des troupes allemandes à Denain, les hauts fourneaux des Forges et aciéries du Nord et de l'Est sont éteints et les usines Cail stoppent tout travail. S'agissant des constructions mécaniques, la plupart des usines sont totalement mises en sommeil, tandis que d'autres connaissent une grande activité. Ainsi, à Raismes, la Franco-belge se spécialise dans la réparation du matériel de chemin de fer et voit son effectif passer de 1500 à 3000 ouvriers pendant l'occupation allemande, mais il semble que les dirigeants de l'usine refusèrent rapidement, "malgré la menace et la promesse de représailles », de continuer ce travail. Le périodique En train nous précise qu'« aucune locomotive, aucun wagon ne seront réparés à Raismes $»^{32}$. L'usine sera apparemment transformée, jusqu'à la fin de la guerre, en une gigantesque scierie. D'autres petits ateliers sont exploités sous administration allemande pour les besoins exclusifs de l'armée d'occupation : c'est notamment le cas des ateliers Malissard-Taza à Anzin. Cependant, l'activité essentielle des territoires occupés du Nord, dès novembre 1914, fut l'extraction charbonnière et, à un degré moindre, les fabrications annexes.

La volonté de liquidation des industries apparait clairement lorsque les Allemands incendient, à la Société française de constructions mécaniques, un magasin des modèles en bois de toutes les machines construites par la firme depuis $1812^{33}$. Même les archives sont détruites. D’autres détériorations résultent de l'emploi des bâtiments comme parc d'artillerie, arsenal de canons, ateliers divers, hôpital, etc. Rappelons qu'aucun des constructeurs de matériel ferroviaire du Nord ne se trouve dans les zones de combat.

31- Odette HARDY-HEMERY, De la croissance à la désindustrialisation..., op. cit., p. 90.

32- En Train, $\mathrm{n}^{\circ} 7$ (revue interne de la firme Alsthom).

33- Odette HARDY-HEMERY, De la croissance à la désindustrialisation..., op. cit., p. 93. 


\section{L'occupation des usines Arbel}

Les troupes allemandes arrivent à Douai le 3 septembre 1914, repartent et reviennent le 4 octobre. Quelques combats ont lieu dans l'usine : un petit groupe de territoriaux français y est tué. «Ces braves furent enterrés à l'endroit où ils étaient tombés ${ }^{34}$. » Mais, globalement, les installations industrielles sont intactes et sont très rapidement occupées par des soldats allemands, qui ont besoin de pain, d'eau et d'espace pour leur logement et celui de leurs chevaux. A Douai, l'occupation commence le 10 octobre 1914 à l'usine $n^{\circ} 1$ par la $1^{\text {re }}$ compagnie bavaroise de boulangers militaires à laquelle se joint, le 14 octobre, la compagnie badoise de réserve du $14^{\mathrm{e}}$ Corps d'armée. L'usine $\mathrm{n}^{\circ} 3$ est occupée le 27 novembre 1914 par une troupe du corps de chemins de fer et 50 chevaux sont logés dans les bureaux administratifs. Pendant la période du $1^{\text {er }}$ octobre au 19 décembre 1914, les Allemands commencent à piller les usines, même si celles-ci sont encore censées appartenir à leurs propriétaires. Sur place et pour faire face, il ne reste que quelques « courageux » employés dont l'ingénieur Falchi et l'agent Godon.

Le 19 décembre 1914, la situation change brusquement ${ }^{35}$ :

«Ce jour-là, un militaire, du nom de Geiger, appartenant à l'inspection civile de la $\sigma^{e}$ armée se présentait chez nous, porteur d'un acte de consignation ainsi libellé :

\section{ACTE DE CONSIGNATION :}

«J'ai consigné aujourd'hui le 19 décembre 1914, aux Établissements Arbel toutes les usines et matériaux se trouvant dans ces établissements, qu'ils lui appartiennent en propre ou à des tiers ». «Le droit de disposer de ces marchandises est réservé dès aujourd'hui à l'administration de l'Armée allemande ; même les personnes faisant partie de cette armée ne pourront en prendre sans la permission de l'autorité soussignée ».

«Le propriétaire et ses employés sont chargés de la surveillance des marchandises consignées ; ils sont avertis des conséquences d’un détournement survenu [...]. »

34- AD Nord 10 R 4563. Dommages de guerre. Société Arbel. «État indiquant les dates d'occupation de nos usines par l'Armée Allemande pendant la guerre. »

35- Ibidem. Société Arbel. Rapport sur les événements survenus aux Établissements Arbel - Usines de Douai pendant la guerre de 1914-1918, dressé par M. Godon, s/agent administratif des Forges de Douai. 22 avril 1919. 
Dès lors, et jusqu'en 1916, les usines Arbel sont occupées en permanence par les troupes allemandes, qui transforment les ateliers tantôt en fabrique d'eau gazeuse, tantôt en hôpital ou en boulangerie. Une fabrique d'eau de Seltz est installée à l'usine no 2 le 3 juillet 1915 et une menuiserie le 18 juillet 1915 , mais pour très peu de temps puisqu'elles sont déplacées respectivement les 7 et 2 août 1915. Le 20 juillet 1915, l'ancienne aubette d'octroi est transformée en écurie. En août 1915, les boulangeries militaires quittent partiellement l'usine pour céder la place à des services sanitaires en vue d'un aménagement des locaux en « ambulance ». En octobre, une nouvelle compagnie de boulangers militaires rejoignit les services d'ambulance installés dans l'usine $n^{\circ} 1^{36}$. Quant à l'usine $n^{\circ} 3$, elle est occupée du 17 au 28 octobre 1915 par la troupe allemande, qui apporte dans les ateliers des canons et une centaine de chevaux environ. Elle a été précédée le 9 octobre 1915 par une maréchalerie composée de deux forgerons et de forges à main s'installant dans l'usine $n^{\circ} 2$. L'usine conserve un minimum d'activité au profit de l'armée allemande : le 11 janvier 1916 quatre voitures de la Société des tramways électriques de Douai y sont amenées pour être transformées en voitures d'ambulance à huit lits et servir ainsi au transport des blessés. Quelques semaines plus tard, le 9 février 1916, les autorités allemandes prennent complètement possession de l'usine $\mathrm{n}^{\circ} 2$ pour y établir un garage pour les voitures de «tramways-ambulances ».

Tout au long de l'année 1916, Arbel voit se succéder des prisonniers de guerre, des soldats allemands, des boulangers. Le 3 mars 1916, 25 prisonniers russes et une escouade allemande s'installent dans l'usine $n^{\circ} 3$. À l'usine $n^{\circ} 1$, la $2^{\mathrm{e}}$ colonne de boulangers de campagne de la réserve de la garde remplace sans interruption, le 9 mai 1916, la compagnie bavaroise de boulangers installée depuis le 5 octobre 1915. L'ancienne aubette d'octroi utilisée comme écurie depuis juillet 1915 est abandonnée dans les premiers jours de mai 1916. La colonne de boulangers de la garde quitte elle aussi l'usine le 24 juillet 1916 dans la nuit. Mais, dès le 24 août 1916 au matin, une nouvelle colonne de boulangers arrive. Entre deux « occupations », le matériel et les locaux sont placés sous surveillance militaire, en attendant l'arrivée de nouveaux contingents.

36- Voir également le récent ouvrage de Roland ALLENDER, 1914-1918 dans le Nord: survivre en pays occupé, Saint-Cyr-sur-Loire, A. Sutton, 2006. R. Allender nous propose en page 42 une carte postale représentant une boulangerie militaire installée dans l'usine Arbel. 
Le passage des soldats allemands s'accompagne en outre d'un comportement coupable. Le laboratoire, mis sens dessus dessous, porte selon l'agent Godon « des traces d'une goujaterie ignoble », tandis que « le magasin d'approvisionnement a été mis dans un désordre abominable $»^{37}$.

\section{Un pillage ordonné et méthodique}

Les conventions de La Haye du 18 octobre 1907 prévoyaient que les prestations en nature ne pouvaient être exigées que pour les besoins de l'armée d'occupation, le paiement devant être fait au comptant ou constaté par des reçus ${ }^{38}$ : à quelques détails près, l'armée allemande respecte ce texte jusqu'en mars 1916. Au cours des deux premières années de la guerre, les réquisitions allemandes portent sur les matières premières ou les produits finis en cours de livraison, l'ensemble des prélèvements étant constaté par des bons. Mais, ensuite, l'autorité d'occupation établit pour toutes les machines utilisables une nomenclature communiquée aux industriels allemands intéressés ${ }^{39}$. L'année 1916 est en effet un tournant dans la guerre : nous passons d'une exploitation abusive à une destruction systématique s'intensifiant progressivement au cours des années 1916 à 1918. Il apparaît aux Allemands que la guerre se livre désormais autant sur le territoire traditionnel des opérations militaires que dans les usines. L'importance prise dans la bataille du matériel de guerre étend donc la guerre d'usure dans le territoire occupé. Dans cette optique, l'occupant juge utile d'apporter des modifications à l'administration du territoire occupé. Après une occupation d'environ deux ans, les autorités allemandes choisissent donc de procéder au démontage de l'outil industriel. Cela commence par la réquisition des matières premières, des produits semifinis, tout ce qui est facilement et rapidement transportable. Puis, dans un second temps, les troupes allemandes « aidées » de prisonniers de guerre russes procèdent au démontage des machines. Le but n'est pas seulement de détruire mais de faire bénéficier l'industrie allemande de cette manne ${ }^{40}$. Ainsi, de nombreux ingénieurs allemands se déplacent à

37- AD Nord 10 R 4563. Dommages de guerre. Société Arbel. Rapport sur les évènements survenus aux Établissements Arbel - Usines de Douai pendant la guerre de 1914-1918, dressé par M. Godon, s/agent administratif des Forges de Douai. 22 avril 1919.

38- Odette HARDY-HEMERY, De la croissance à la désindustrialisation..., op. cit., p. 93.

39- Il s'agit du document déjà cité : L'Industrie en France occupée, traduit et publié en France en 1923.

40- Parallèlement, en avril 1916, certains équipements, en commençant par les cylindres de laminoirs, sont directement mis à la mitraille. 
Douai pour faire leur choix. C'est alors que le caractère économique de ces prélèvements saute aux yeux. L'épouse d'un officier allemand déclare à Falchi et Godon que l'Allemagne étant entourée d'ennemis, elle est bien obligée de prendre par ailleurs ce qu'elle ne peut plus se procurer. Mieux, les Allemands s'assurent également de l'élimination d'un concurrent dangereux : la réquisition en mai 1917 de deux presses de 1200 tonnes, dont l'une de 22 mètres de long, ne pouvant se prêter à la fabrication du matériel de guerre, est liée à la perte avant le conflit d'un marché pour la fourniture de wagons destinés à la Roumanie par une entreprise allemande que nous avons signalée : «c'est avec cet engin que vous nous avez enlevé la commande de 100 wagons pétroliers roumains, nous allons l'emporter dans nos usines et c'est nous qui ferons les wagons Arbel à votre place ${ }^{41}$.»

En 1917, les Allemands accélèrent leurs dévastations. Le 25 février, une nouvelle compagnie de boulangers militaires, la réserve Bäckerei Kol 3 arrive à l'usine $n^{\circ} 1$ avec douze fours roulants. Le 21 mars, un parc de génie suit avec un matériel considérable remisé dans tous les bâtiments de l'usine $n^{\circ} 3$. En avril, en plus des services déjà installés, de forts contingents de troupes (compagnies ou bataillons) sont cantonnés dans l'usine $n^{\circ} 1$, avec voitures et chevaux logés pêle-mêle à côté des machines-outils. En mai, de nouvelles équipes allemandes arrivent et accélèrent l'allure des chargements, l'enlèvement des machines et la destruction d'une partie d'entre elles. Le 25 juillet 1917, de nouvelles équipes allemandes appartenant à l'entreprise Gutehoffnungshütte, d'Oberhausen, arrivent à l'usine $\mathrm{n}^{\circ} 1$ avec des soldats du service d'aviation et procèdent au démontage des cinq halls du wagonnagemontage. Dans le courant du mois d'août 1917, elles continuent de détruire presque toutes les machines ou appareillages restants, tels que marteaux-pilons, machines à vapeur, fours à réchauffer et à recuire, machines-outils diverses, ponts roulants électriques, générateurs, chaudières, gazogènes, appareillages d'épuration et du service d'eau, machines à meuler. Les bâtiments de la forge et de l'usine $\mathrm{n}^{\circ} 2$ sont également en voie de démontage. En septembre 1917, le démontage des bâtiments du wagonnage-montage et de l'usine $n^{\circ} 2$ est terminé tandis que se poursuit la démolition du bâtiment de la forge. À l'usine $\mathrm{n}^{\circ}$ 3, les deux grands fours Martin de $30 \mathrm{t}$ et leurs annexes, le laminoir à grosses tôles, un générateur de secours et tous les fours sont complètement détruits. En octobre 1917, la démolition du bâtiment de la forge (usine $n^{\circ} 1$ ) est terminée. De nouveaux ponts roulants et des

41- La presse de 22 mètres est retrouvée après la guerre et récupérée par Arbel. 
chabottes de pilons sont détruits. Le 18 octobre 1917 a lieu le départ d'une partie des prisonniers russes, le 22 celui de l'équipe Naugebauer et le 30 celui de la colonne de boulangers. Il reste toutefois à l'usine un four pour la cuisson du pain blanc et sept militaires pour la garde des matériaux laissés par le service de subsistance. À l'usine $n^{\circ} 3$, le démontage du bâtiment des gazogènes est achevé. Le parc à lingots, le bâtiment des tôleries et le bâtiment des fours Martin sont démontés. En novembre 1917, le bureau de montage et le magasin (usine $n^{\circ} 1$ ) longeant la route d'Aniche sont démolis, le bâtiment des roues et bandages, le hall de coulée et le parc à mitrailles démontés (usine $n^{\circ} 3$ ). En décembre 1917, les machines-outils de l'usine subissent le même sort : raboteuses, mortaiseuses, aléseuses, tours à essieux montés, ponts roulants, etc. Le démontage des bâtiments de l'usine $n^{\circ} 3$ se poursuit : cette fois, c'est au tour des bâtiments des tôleries, des roues et bandages, des fours Martin, des bureaux d'ateliers, des grands bureaux de la direction, des magasins et de la station centrale électrique.

L'année 1918 voit la poursuite des enlèvements : tours à essieux, coudes, mortaiseuses, ponts roulants, enlèvement des charpentes métalliques des bâtiments déjà démontés... Les dernières parties métalliques des bâtiments des tôleries, des roues et bandages, des bureaux, du magasin et de la station électrique sont chargées sur des wagons en vue de leur transfert en Allemagne. En avril 1918, les Allemands procèdent à l'enlèvement des archives du sous-sol, à la destruction des meubles classeurs et d'une partie des archives. Le reste des documents est réuni en gros ballots, chargé sur des wagons pour partir aussitôt vers une « destination inconnue ». En mai 1918, les derniers ballots d'archives sont déposés sous l'atelier d'ajustage, en attendant leur départ, mais exposés aux intempéries. Le $1^{\text {er }}$ mai, les bâtiments et locaux de l'usine $n^{\circ} 1$ sont transformés en morgue pour des corps en provenance du front... L'occupation et le pillage cessent le 17 octobre 1918, date de l'évacuation allemande.

\section{Les bons de réquisition : la résistance s'organise chez Arbel}

Les employés et ouvriers mobilisés, il ne reste dans les usines et ateliers que peu de personnel : quatre ou cinq personnes à Douai, un peu plus à Denain, où nous trouvons le directeur général, Auguste Thomas, et le directeur des ateliers, Rebourg. Guère plus sans doute à Fives, à Crespin, à Marpent... Ce «personnel » demeure à son poste avec l'unique souci de protéger les installations contre les déprédations de l'ennemi ne procèdant d'abord qu'à des réquisitions secondaires, 
mais ses protestations réitérées ne servent à rien : "Nous avons des ordres à exécuter; nous n'avons pas de matières premières et nous devons en prendre où il en reste. Protestez, protestez tant que vous voudrez mais rien n'y fera ${ }^{42}$. » Les employés veillent donc à ce que des bons de réquisition réguliers soient rendus en échange de chaque objet pris. La tâche n'est pas aisée, car les enlèvements se font souvent en vrac. Falchi, chef de l'emboutissage, seul membre de la direction à être resté à Douai, sera d'ailleurs détenu par les Allemands à la prison de Cuincy pendant sept mois (à partir du 31 mars 1917). Les Allemands ont cependant parfaitement, et dès le début de l'occupation, établi la façon dont les choses doivent se passer. L'acte de consignation des Usines Arbel en date du 19 décembre 1914 précise que ${ }^{43}$ :

« À l'enlèvement de ces marchandises, on remettra à l'autorité allemande 3 factures portant l'indication exacte de la quantité et de la qualité des marchandises et de leurs prix ; la fixation définitive des prix sera faite ultérieurement en Allemagne.

"L'indication des quantités doit correspondre aux poids bruts ou quantités constatés au moment de l'enlèvement. La partie prenante, signera l'une des factures en ajoutant la formule dont voici la traduction française : poids bruts ou quantités vérifiés. Nom, grade, formation de troupe du preneur, lieu, date.

«Cet exemplaire restera entre les mains du propriétaire ou de son remplaçant comme quittance provisoire.

"Il sera échangé plus tard par l'autorité sous-signée contre exemplaire, signé et cacheté par elle, qui tiendra lieu de bon de réquisition. »

Les Allemands scindent les réquisitions en deux grandes catégories : la première comprend les matériaux utilisés sur le front (non remboursables), la seconde les matériaux expédiés en Allemagne (remboursables). Partant de ce principe, ils n'ont que peu de chose à payer, la majeure partie des réquisitions, à cette époque, ayant été faite pour les troupes de combat. Impuissants face à la ruine progressive des usines, les employés conservent une attitude énergique et emploient tout leur temps à surveiller les prélèvements et à réclamer des bons de réquisition. C'est d'ailleurs la seule tâche que leur laissent les Allemands. Cependant, au risque d'encourir les pires représailles, le personnel lutte

42- AD Nord 10 R 4563. Dommages de guerre. Société Arbel. Rapport sur les évènements survenus aux Établissements Arbel - Usines de Douai pendant la guerre de 1914-1918, dressé par M. Godon, s/agent administratif des Forges de Douai. 22 avril 1919

43-Idem. 
jusqu'au bout contre les irrégularités : les agents de Cail obtiennent ainsi plus de 2000 bons de réquisition et récupèrent une partie du matériel volé en montant sur les wagons chargés, aux heures des repas, pour en retirer tout ce qui a été accumulé sans réquisition, parviennent à éviter la dispersion complète des archives en dissimulant les pièces de comptabilité dans des caches!

La situation est identique chez Arbel, chez Baume-Marpent où les employés notent les sorties des usines, ce qui, au lendemain de l'Armistice, simplifiera grandement la tâche des ingénieurs recherchant dans le Reich l'outillage enlevé. Mieux, les agents d'Arbel notent les noms des civils et des entreprises venus faire leur choix dans les ateliers ${ }^{44}$ ! À Marpent, on prend soin de consigner la destination des wagons. Il est d'ailleurs à remarquer qu'à Douai des visites de civils ou de militaires précèdent presque toujours les enlèvements importants. Bien organisées, ces visites sont menées par un bureau militaire, attaché en permanence aux usines, ayant pour rôle de contrôler et de réglementer les réquisitions.

Les bons de réquisition portent finalement les prélèvements chez Arbel aux montants suivants (tabl. 3).

Tableau 3. Montant des réquisitions dans les Usines Arbel 1914-1918

\begin{tabular}{|c|c|}
\hline Périodes & Montant des réquisitions \\
\hline Octobre à décembre 1914 & $93 \mathbf{7 3 8 , 1 8}$ \\
\hline 1915 & 2880248,90 \\
\hline 1916 & 5038703,40 \\
\hline 1917 & 10173156,24 \\
\hline 1918 & 13683461,15 \\
\hline Total & $\mathbf{3 1 8 6 9 3 0 7 , 8 7}$ \\
\hline
\end{tabular}

44- Civils et entreprises : Mannesmann fr. fabricants de tubes à Remscheid ; Fonderies de Dillingen, à Dillingen-Saar (Peters Gross) ; Vulcan Werke à Hambourg (Naugebauer) ; Concordia Hutte à Engers (Funguitz) ; Thyssen et Cie à Hagondange (Alsace) et Mulheims-Ruhr; Dinglersche maschin. à Zweibrucken (Wimberg); Press et Hammer Werke à Brackwerde; Adolf Schwinn à Hambourg (Dell); Krupp à Essen (Peters Emsters); Patrone à Dusseldorf (Rickmann, Kaps, Grosse); Henchel à Hattingen (Gotitz), etc. 
Le tableau 3 montre que, à partir de 1917, les Allemands accélèrent leurs dévastations. Même constat à Crespin où les prélèvements à la Société de Blanc-Misseron se font «avec une recrudescence marquée dès le commencement de 1917 après l'échec des propositions de paix de l'Allemagne $»^{45}$. L'année 1918 correspond à l'enlèvement des bâtiments; le montant de 1918 est dû à cette particularité et aussi à la régularisation d'une quantité de réquisitions de 1917. Aux usines de Baume-Marpent, les réquisitions s'opèrent à la même cadence pour former au total vingt bordereaux de bons de réquisitions pour la seule usine de la rive gauche ${ }^{46}$. Au départ, ces bordereaux et ces bons, établis juste avant le départ des matériaux ou des machines, lorsque la réquisition devient "effective », doivent être adressés aux autorités militaires allemandes pour paiement du préjudice. Ils servent finalement à l'établissement des dossiers de dommages de guerre à l'issue du conflit. L'importance de ces bons pour les industriels est telle que la firme Arbel ${ }^{47} \mathrm{~s}^{\prime}$ empresse, après les avoir récupérés, de les placer dans un coffrefort à Paris !

\section{Le repli partiel des activités à Couzon}

Pendant l'occupation de Douai, la Société Arbel ne reste pas désœuvrée. Elle utilise ses ateliers de Couzon à Rive-de-Gier, dont Lucien Arbel prend la direction en octobre $1915^{48}$. De leur côté, Fives-Lille réactive ses ateliers de Givors et la Société Malissard-Taza et la Boulonnerie de Thiant créent dès 1917 à Saint-Denis une usine de finissage d'obus qui se transforme, aussitôt la guerre achevée, en boulonnerie ${ }^{49}$. Le groupe Empain et les usines de Jeumont replient leurs activités du Nord vers la région parisienne, dans l'usine de la Plaine Saint-Denis (usine qui deviendra, par la suite, la division appareillage électrique du groupe).

45- Archives ANF. Société de Blanc-Misseron. Assemblée générale du 17 décembre 1919 pour les exercices 1914-1915 à 1918-1919.

46- AD Nord 10 R 4664. Dossier de dommages de guerre. Société Anonyme de construction de Baume et Marpent. Il s'agit de la partie de l'usine située sur la rive gauche de la Sambre, en l'occurrence l'aciérie.

47- ANC (CAMT) 70 AQ 6. Établissements Arbel. Conseil d'administration. 8 février 1919.

48- ANC (CAMT) 70 AQ 5. Établissements Arbel. Conseil d'administration. 28 octobre 1915 .

49- Odette HARDY-HEMERY, De la croissance à la désindustrialisation..., op. cit., p. 93. 
À Couzon, la fabrication de munitions est organisée malgré un gros problème de main-d'œuvre. Dans un premier temps, la firme accueille des prisonniers polonais puis, en janvier 1917, elle obtient l'attribution par le gouvernement français de 200 ouvriers marocains, pour lesquels est organisé un cantonnement ${ }^{50}:$ dortoir, lavabos, cantine, café maure, salle de police et prison! Mais ils s'avèrent difficiles à gérer. Suite à un « incident »- le décès de six d'entre eux « dû à la rigueur de la température »-ils refusent de continuer le travail à l'extérieur. Qu'à cela ne tienne : 80 Marocains sont renvoyés aux autorités militaires et au dépôt de Marseille et échangés contre un nouveau contingent. Parallèlement, les administrateurs se posent la question de savoir comment attacher cette main-d'œuvre en France ? peut-être par la création de « colonies marocaines » ? Les travailleurs d'Afrique du Nord ne sont pas les seuls à causer des soucis. En septembre 1917, un dénommé Jobard, chauffeur de chaudière, est reconnu responsable de l'explosion de sa machine par «manque de surveillance volontaire $»^{51}$. L'accident est signalé au contrôle de la main-d'œuvre et l'ouvrier renvoyé au front. Quelques mois plus tard, du 21 au 26 mai 1918, un mouvement de grève, dont « la source antipatriotique a été nettement définie », affecte les usines de Rive-de-Gier, suite à un mouvement général décidé par les syndicats métallurgistes ouvriers de la région de la Loire pour protester contre le rappel des ouvriers atteints par la loi du 10 août 1917 et demander la paix. Plusieurs des ouvriers de Rive-de-Gier, inculpés d'atteinte à la liberté du travail, sont alors arrêtés ou envoyés au front.

Mais le grand souci de la firme Arbel est le sort de Luc Arbel, l'héritier, mobilisé lui aussi et dont l'absence de nouvelles fait « frémir » tout le conseil d'administration.

\section{Le constat des dégâts}

La fin de la Grande Guerre s'achève tragiquement pour Arbel et les constructeurs ferroviaires du Nord de la France. Leurs usines ont quasiment disparu, ne laissant derrière elles que des ruines, des machines éparpillées, des ouvriers dispersés ou morts sur les champs de bataille. La guerre laisse des plaies profondes. Les dévastations concernent l'ensemble du tissu industriel. Dans le Valenciennois, « $90 \%$ des immobilisations lourdes accumulées en un siècle et demi sont

50- ANC (CAMT) 70 AQ 6. Établissements Arbel. Conseil d'administration. 13 janvier, 10 février, 10 mars et 14 avril 1917.

51- Idem, 8 septembre 1917. 
détruites $»^{52}$. L'essentiel des installations charbonnières est anéanti. Aux ateliers de la Société de Blanc-Misseron ${ }^{53}$, les soldats allemands font exploser les installations et incendient ce qui reste dans les quelques jours qui précèdent leur départ, en octobre 1918. Seul le bâtiment des bureaux est à peu près épargné. En octobre 1918, à Denain, quelques jours avant l'arrivée des soldats canadiens, les Allemands finissent leur travail de destruction et scient les piliers supportant les bâtiments restés debout. On abat aussi les derniers ponts roulants demeurés en place. En quatre ans, un mois et vingt-cinq jours, les Allemands ont enlevé des ateliers Cail $24000 \mathrm{~m}^{2}$ de surface couverte et 50000 tonnes de matériel ; 3000 wagons chargés et un nombre incalculable de camions ont servi à la besogne. Sur les bâtiments subsistants, $10000 \mathrm{~m}^{2}$ sont absolument hors d'usage, les 24000 autres gravement abîmés.

À Douai, aux usines Arbel, de rares ateliers sont encore debout et en très mauvais état. Quant à l'outillage, il a totalement disparu : "après quatre années de guerre, nous trouvons nos terrains dans l'état où nous les avons achetés il y a 25 ans $^{54}$. » Cependant, Lucien Arbel précise que les «métallos» de Douai ne rechignent jamais à effectuer par tous les temps et dans des conditions souvent pénibles des travaux de déblaiement, de terrassement, de couverture, de vitrerie qui permettent le redémarrage des usines dans les délais les plus réduits ${ }^{55}$.

Pour les industriels du Nord de la France, le sentiment de revanche sur l'Allemagne est alors dominant. L'Allemand doit payer : pour les usines saccagées, ruinées, pour ses crimes. La Société Arbel réclame donc « justice ». L'année 1918 se termine par la visite dans le Nord Pas-de-Calais de Louis Loucheur, ministre des régions libérées, qui laisse espérer le passage du président américain Wilson à travers les régions dévastées du Nord de la France. En fait, ce voyage n'aura pas lieu et sera remplacé par une visite, en mars 1919, des journalistes attachés à la Conférence de la Paix. Une lettre de la firme est cependant adressée à T.W. Wilson en décembre 1918 : «Ceci est odieux, intolérable, inadmissible, plus odieux, plus intolérable que toutes les misères des

52- Odette HARDY-HEMERY, De la croissance à la désindustrialisation..., op. cit., p. 94.

53- Archives ANF. Société de Blanc-Misseron. Assemblée générale ordinaire. 4 juillet 1919 .

54- AD Nord 10 R 4564. Dossier des dommages de guerre. Société Arbel. Lettre des Établissements Arbel au président Wilson le 18 décembre 1918.

55- La Passerelle, $\mathrm{n}^{\circ}$ 12, novembre 1957. 
combats $^{56}$. » Pour Arbel, les alliés doivent imposer à l'Allemagne le coût de la guerre et des destructions afin de permettre à toutes les régions envahies des pays occupés alliés un redressement économique plus rapide. C'est aussi le souhait du président de la SFCM, Louis Le Châtelier. Plusieurs possibilités sont offertes aux industriels. La première solution est de saisir chez l'ennemi vaincu ce dont on a besoin pour la reconstruction : «le droit pour les alliés de s'approprier chez l'ennemi des biens privés, en considération seulement de la valeur d'utilisation qu'ils en pourraient retirer, leur est donc ouvert sans limite ${ }^{57}$. Pour Le Châtelier, «prendre ou reprendre chez l'ennemi de l'outillage ou des matières premières, et les livrer à qui a été dépossédé de l'équivalent, c'est l'opération simple qui se présente à l'esprit, dès qu'on aborde la question. Pratiquée largement, elle fournira un élément de solution, offrant de l'importance pour certaines industries, la mienne principalement, mais rien qu'un élément [...] ». Effectivement, puisque l'Allemagne doit payer, se profile un projet de reprise des usines allemandes d'Alsace-Lorraine par des associations d'industriels sinistré $\mathrm{s}^{58}$, composées de métallurgistes et mécaniciens... Mais c'est surtout de l'argent qu'il faut, et il s'agit de sommes considérables.

\section{Le rôle de l'État et les dommages de guerre}

Avant même la fin de la guerre, dès septembre $1918^{59}$, les ingénieurs de la firme mènent différentes études sur son avenir : quelle est la meilleure voie pour le développement des Établissements Arbel ? Fautil reconstruire les ateliers de Douai ? Ou choisir une autre région pour y constituer une nouvelle usine, comportant des hauts fourneaux et fours à coke, ou alimentée par la force électrique provenant de chutes d'eau ? La dualité du type de reconstruction, en l'état ou modernisation profonde, se retrouve d'ailleurs plus ou moins dans toutes les entreprises.

Arbel décide finalement de rester dans le Nord. La reconstruction peut commencer. En fait, les débouchés ne font pas défaut, ni les commandes aux entreprises, mais le manque de matières premières, de tôles, de machines ne favorise pas la reprise des activités. Le rythme du relèvement des usines est donc inégal. Il faut parer au plus pressé :

56- AD Nord 10 R 4564. Dossier des dommages de guerre. Société Arbel. Lettre des Établissements Arbel au président Wilson. 18 décembre 1918. Cette lettre est jointe en annexe. La Passerelle, $\mathrm{n}^{\circ}$ 13, janvier 1958.

57- Louis LE CHATELIER, Sowvenirs, 1873-1923, Paris, impr. Chaix, 1924, 194 p., p. 6-7 et 9. 58- AN (CAMT) 70 AQ 6. Établissements Arbel. Conseil d'administration. 8 février 1919. 59- Idem, 14 septembre 1918. 
réaménager l'habitat et faire redémarrer l'industrie. La société entreprend de nettoyer les sites dès le départ des Allemands en octobre 1918. Le 9 novembre, l'administrateur-délégué de la Société Arbel expose son programme de reconstruction des usines « en vue d'organiser, dans le plus bref délai possible, des fabrications permettant de donner du travail à notre personnel et à la population douaisienne, tout en répondant aux nécessités du moment, au point de vue national $»^{60}$.

Le projet de reconstruction des usines de Douai comporte trois points :

1. Fabrication des charpentes métalliques. Le matériel nécessaire à cette fabrication, initialement acheté pour être installé à Couzon, sera transporté à Douai, dans l'ancien atelier d'emboutissage, pendant la durée de construction des nouveaux ateliers. Un minimum de production de 3000 à 3500 tonnes par an est envisagé.

2. Fabrication des essieux montés. Les outillages nécessaires seront prélevés aux usines de Couzon, où ils ne sont pas utilisés. Une installation provisoire sera faite à Douai dans l'ancien atelier de finissage des essieux coudés. Les roues seront fournies ébauchées par les ateliers de Couzon, tandis que les bandages seront achetés à l'extérieur comme avant la guerre. Quant au finissage, il sera installé dans l'ancien atelier d'ajustage à Douai au moyen de tours en partie disponibles à Couzon.

3. Réparation du matériel roulant. Des abris provisoires devront être construits pour permettre d'entreprendre la réparation de matériel roulant.

Ce programme provisoire est réalisé au fur et à fur mesure des disponibilités de la Société Arbel et des dédommagements versés par l'État, qui avançe immédiatement $30 \%$ des estimations des dommages de guerre, et des banques. En effet, afin de remédier aux différents dommages, immobiliers et mobiliers, l'État a adopté rapidement des mesures pour remettre le pays en marche. De nombreuses structures sont créées et toute une série de lois et de décrets sont promulgués pendant et après la guerre ${ }^{61}$.

60- Id., 9 novembre 1918.

61- Cf. René ROMAN (dir.), Code des dommages de guerre. Lois et décrets. Circulaires et instructions ministérielles. Décisions de jurisprudence, Paris, Revue des dommages de guerre, Collection Roman, 1924, 259 p. 
- 26 décembre 1914 : loi ouvrant un premier crédit de 300 millions pour la réparation des dommages matériels résultant des faits de guerre ; création du comité consultatif des dommages de guerre et des commissions d'évaluation ;

- 1915 : création du guide du sinistré qui doit permettre à chaque sinistré de connaître les démarches afin d'être indemnisé rapidement et efficacement ;

- juillet 1916 : décret réunissant un comité de ministres et d'architectes pour établir des normes de reconstructions ;

- août 1916 : création du Comptoir central d'achats industriels (CAA) ;

- 1917 : création de l'Office de reconstruction industrielle (ORI) et de l'Office de reconstruction agricole (ORA);

- avril 1917 : loi des processus des sinistrés instaurant trois catégories de dommages liés à la guerre : réquisitions et dommages pécuniaires ( $1^{\text {re }}$ catégorie), dommages immobiliers ( $2^{\mathrm{e}}$ catégorie) et mobiliers ( $3^{\mathrm{e}}$ catégorie) ;

- 17 avril 1919 : loi sur la répartition des dommages de guerre entre les différentes régions et activités sinistrées.

L'attribution des dommages de guerre est subordonnée pour une part au réemploi, c'est-à-dire essentiellement à la construction d'une usine pouvant se situer dans un rayon de 50 kilomètres par rapport au sinistre $^{62}$. Pour prétendre à une indemnité de reconstruction, il faut également déposer un dossier instruit par les commissions cantonales pour les petits dommages et, au-dessus d'un million de francs, par le comité central de préconciliation. Le constat des dommages est établi par un ingénieur désigné pour chaque secteur. Cette procédure assez lourde entraîne des longueurs d'autant que, presque systématiquement, les dossiers très importants sont revus à la baisse, ce qui entraîne toute une série d'appels en recours. D’ailleurs, le 19 mars 1919, Pierre Arbel, estimant le préjudice à plus d'une centaine de millions, demande la désignation immédiate d'un expert conformément à la loi du 5 juillet 1917. Il attend avec impatience, devant l'urgence des travaux à entreprendre, l'octroi d'une avance sur indemnités. Effectivement, dans tous les cas, en attendant les décisions, des avances en numéraire ou en

62- Odette HARDY-HEMERY, De la croissance à la désindustrialisation..., op. cit., p. 102103. 
nature sont faites par l'État sur justificatif de dépenses ${ }^{63}$. Il s'agit de parer au plus pressé et de permettre aux entreprises concernées de commencer à reconstruire.

Les dommages subis par les Forges de Douai donnent lieu au dépôt d'une première demande d'indemnité auprès de la commission cantonale pour une somme de 39407 672,80 francs. En 1923, le seul montant des factures des marchés de déblaiement des usines de Douai s'élève à 2640 744,08 francs! Ce qui est sûr, en tout cas, c'est qu'Arbel obtient des avances lui permettant de reconstruire assez vite et que l'évaluation définitive dure plus d'une décennie. Le $1^{\text {er }}$ mai 1930, une note manuscrite jointe au dossier de dommages de guerre précise que les indemnités versées au crédit des Établissements Arbel s'élèvent à 98439145 francs, montant qui semble définitif ${ }^{64}$.

Pourtant, dès 1920, l'État fait difficilement face aux demandes des entreprises : une série de combinaisons financières est alors échafaudée par les sinistrés eux-mêmes pour se procurer des fonds. Une solution consiste à procéder à une augmentation de capital ou, plus fréquemment, à faire appel à un emprunt obligataire. Les industriels vont très vite se regrouper et émettre des emprunts gagés sur les certificats d'indemnités de leurs commettants. Sur le plan national se créent divers organismes: le Comptoir central d'achats industriels pour les régions envahies (CAA), dont Louis Le Châtelier est le vice-président, le Groupement pour la création du Crédit national pour faciliter la réparation des dommages causés par la guerre... Le CAA constitue des stocks afin de prévenir le danger d'une inflation des prix industriels, puis travaille en liaison étroite avec le ministère de la Reconstruction, qui divise les régions libérées en dix secteurs. L'ampleur de la tâche oblige très vite le CAA à décentraliser ses structures et une agence est mise sur pied dans chaque secteur. Les Établissements Arbel adhèrent à l'Association des usines métallurgiques sinistrées du Nord et de l'Est ${ }^{65}$. Dès 1921, ils passent une convention de paiement avec l'État et délèguent leurs titres d'annuité au groupement de la grosse métallurgie, lequel émet différents emprunts. Quant à la SFCM, elle intègre l'Association pour la reprise de l'activité dans les régions envahies. Elle crée également, avec FivesLille et Thomson-Houston, la Société de reconstitution d'usines sinistrées. D’autres structures sont créées au début de l'année 1919 par le Comité

63- La sous-série $10 \mathrm{R}$ (consultée aux AD Nord) contient les dossiers des dommages de guerre : instruction, vérification, et paiement des avances aux sociétés.

64- AD 10 R 4565. Dossier des dommages de guerre. Société Arbel. Note manuscrite du $1^{\text {er }}$ mai 1930.

65- AN (CAMT) 70 AQ 6. Établissements Arbel. Conseil d'administration. 8 février 1919. 
des forges de France en vue de la répartition des commandes et des matières premières : le Comptoir sidérurgique de France, le Comptoir des tôles et larges plats et le Comptoir des fers et aciers marchands ${ }^{66}$.

\section{La reconstruction des usines}

La reconstruction démarre très vite. En novembre 1918, il reste chez Arbel, à l'usine 1 (boulevard Faidherbe), quelques bâtiments encore debout. Ce sont les nefs principales (plus des appentis et annexes) des halls d'emboutissage, hall d'ajustage et bâtiment d'aciérie, et les magasins, la loge du concierge et l'immeuble d'administration. Rappelons que, juste avant la guerre, l'usine 1 comportait l'emboutissage, la forge, l'aciérie, la station centrale, l'ajustage, le wagonnage... Dès le début 1919, les travaux commencent. Le hall d'emboutissage est démonté et reconstruit sur le terrain de l'usine 3 , entre les halls des fours et le cimetière. Le hall d'ajustage est ripé et déplacé pour constituer l'atelier de mécanique. Quant au bâtiment d'aciérie, il est démonté et mis au parc de l'usine 3. Les matériaux qui le constituent servent à édifier les magasins et bâtiments d'importance secondaire, soit à l'usine 3, soit à l'usine 5 . À la place de ces bâtiments, entre 1919 et 1923, sont reconstruits les cinq halls, le parc aux fers et le château d'eau. Le bâtiment de la tôlerie fine est monté en 1927 et 1928. À l'usine 2 (sise rue Giroud) existaient deux halls remplacés en 1918 et 1923 par un seul.

L'usine 3, établie sur les terrains de la Berce Gayant, comportait avant guerre les constructions suivantes : laminoirs, roues et bandages, hall de coulée, hall de fours, parc à mitraille, gazogène, etc. L'usine, neuve en 1914, ne comporte plus en novembre 1918 que le hall de coulée, le hall des fours, le bâtiment d'entrée et le parc à mitrailles. À la place de ces bâtiments et dans leur voisinage immédiat sont construits entre 1918 et 1923 six halls, la station centrale, le gazogène et deux travées de 13 mètres de halls de coulée et des fours. Le bâtiment d'emboutissage provenant de l'usine 1 avant guerre est remonté entre le parc à mitrailles et le cimetière. En octobre 1919 la Compagnie du Nord donne son accord pour que soit reconstruite la passerelle qui, enjambant les voies et les quais de la gare de Douai, permettait la jonction entre les ateliers ${ }^{67}$. Deux autres usines sont construites dans la foulée de la reconstruction par les Établissements Arbel. Il s'agit des usines 4 et 5 . L'usine 4 , installée près du pont de Lille, sur la rive droite de la Scarpe, sert quelque temps à la réparation et à la construction de wagons. Elle

66- Ibid.

67- La Passerelle, $\mathrm{n}^{\circ} 13$, janvier 1958. 
comporte trois bâtiments et un hangar métallique, le tout sur 35000 $\mathrm{m}^{2}$. Il s'agit d'importants bâtiments américains achetés au camp de Beauvoir. Mais en raison de la pénurie des commandes, les activités sont transférées à l'usine 1. L'usine 4 est revendue à la Compagnie des mines d'Aniche, dès janvier 1926, pour six millions de francs ${ }^{68}$.

L'usine 5, installée boulevard Vauban, sert à la fabrication des péniches. Elle est construite d'une part entre 1922 et 1923, d'autre part, en 1926, 1927 (couverture des cales) et 1929 (atelier). La partie édifiée entre 1922 et 1923 comprend un bâtiment servant de bureau au contremaitre, de logement au concierge, d'atelier et de salle de compresseur, ainsi que les clôtures. Il a été réalisé avec des matériaux (fer, bois, tôles ondulées) provenant des ruines du bâtiment de l'aciérie d'avant guerre de l'usine 1 . Il est à noter, cependant, qu'Arbel annonce que ces réparations ont demandé à peu près uniquement de la main-d’œuvre et « les charpentes ont pu être remises en état sans apport de pièces métalliques nouvelles, tout au plus a-t-on dû fournir des pièces d'assemblage, boulons et rivets en quantité que nous évaluerons forfaitairement à 2 tonnes ».

Au lendemain de l'Armistice, c'est finalement à une mutation organisationnelle qu'on assiste aux Usines Arbel. Avec la guerre, les aciéries n'existent plus, les hauts fourneaux et les fours Martin sont détruits, et Arbel ne les reconstruit pas. L'entreprise reçoit du fait de la guerre une orientation différente: en 1920, elle abandonne ses fabrications annexes pour se consacrer à l'emboutissage et au wagonnage. Seconde rupture : la modernisation et l'expansion accompagnent la reconstitution. Les Forges de Douai augmentent leur capital et font reconstruire des usines plus vastes (on ne se soucie pas de recréer les surfaces détruites dans l'état d'avant 1914), ce qui ne correspond pas au schéma de reconstitution à l'identique proposé souvent pour les industries du Nord. Globalement pourtant, dès 1922, la reconstruction est achevée. À l'inverse, la SFCM (Cail) reconstruit ses usines de Denain en utilisant les fondations des bâtiments de 1914: chaque société est un "cas d'espèce $»^{69}$. Pour l'entreprise Arbel, c'est cependant un essai modéré de reconversion: elle conserve sa personnalité industrielle basée sur l'emboutissage.

68- AN (CAMT) 70 AQ 90. Forges de Douai. Vente de l'usine 4 à la Compagnie des mines d'Aniche.

69- Odette HARDY-HEMERY, « La reconstruction industrielle après la première guerre mondiale ", in Jean Favier (dir.), Reconstructions et modernisation : la France après les ruines : 1918, 1945, Paris, Archives nationales, 1991, p. 137. 


\section{Sources d'archives}

Les éléments les plus riches sont apportés par les dossiers de dommages de guerre (sous-série $10 \mathrm{R}$ des archives départementales). Très différents d'une société à l'autre, les dossiers se composent d'informations sur l'outillage des ateliers, les démolitions, les pillages : ils ont permis à l'époque d'instruire, de vérifier et de payer les avances auprès des sociétés. Dans le cas d'Arbel-Douai, les dossiers fournissent notamment des albums photographiques, un historique très précis de l'occupation des usines de Douai sur la période 1914-1918 et la lettre adressée au président des États-Unis, T. W. Wilson («Justice », 191970). Nous n'en proposons pas un inventaire détaillé, car les documents y sont insérés sans aucun souci de méthode. Précisons simplement que la cote $10 \mathrm{R} 4563$ contient un document exceptionnel qui permet de suivre de façon très précise l'occupation et le pillage des Forges de Douai : «Rapport sur les événements survenus aux Établissements Arbel Usines de Douai pendant la guerre de 1914-1918, dressé par M. Godon, s/agent administratif des Forges de Douai. 22 avril 1919 »(42 pages) ainsi qu'un «État indiquant les dates d'occupation de nos usines par l'Armée Allemande pendant la guerre. »

\section{Archives départementales du Nord}

Série R (Affaires militaires). Sous-série $10 \mathrm{R}$

\section{Dossiers de sinistrés :}

10 R 4563 Établissements Arbel. Forges de Douai, Forges de Couzon.

10 R 4564 Établissements Arbel. Forges de Douai.

10 R 4565 Établissements Arbel. Forges de Douai.

10 R 4664 Société anonyme de construction de Baume et Marpent, à Marpent.

10 R 4697 Société franco-belge. Matériel de chemins de fer.

\section{Tribunaux de dommages de guerre :}

10 R 5492 Ateliers de construction du Nord de la France, à Blanc-Misseron.

70- Cf. annexe. 


\section{Archives nationales, Centre des archives du monde du travail}

Les archives de la firme Arbel ont également apporté des éléments précis. Les cotes suivantes ont été compulsées :

\section{AQ Établissements Arbel}

70 AQ 1 Procès-verbaux du conseil d'administration.1894-1901.

70 AQ 2 Procès-verbaux du conseil d'administration. 1901-1906.

70 AQ 3 Procès-verbaux du conseil d'administration. 1907-1910.

70 AQ 4 Procès-verbaux du conseil d'administration. 1910-1912.

70 AQ 5 Procès-verbaux du conseil d'administration. 1912-1916.

70 AQ 6 Procès-verbaux du conseil d'administration. 1916-1919.

70 AQ 90 Actes constitutifs de la société. Contrats divers. 18941928.

Ainsi que :

\section{AQ Compagnie du Nord}

202 AQ 1267 Livraison du matériel par les constructeurs (18671930).

\section{Archives privées}

Nous avons, enfin, utilisé divers documents provenant des archives de la firme ANF, aujourd'hui Bombardier à Crespin (Nord), et de la chambre syndicale des fabricants et constructeurs de matériel de chemin de fer, devenue la Fédération des industries ferroviaires (FIF, à Paris). Il s'agit de :

- ANF - Société de Blanc-Misseron : Rapports présentés aux assemblées générales des 4 juillet 1919 et 17 décembre 1919.

- ANF : Rapport présenté à l'assemblée générale ordinaire du 10 novembre 1923.

- Chambre syndicale : Rapport présenté à l'assemblée générale ordinaire du 17 juin 1918 (fonctionnement de la chambre syndicale pendant l'année 1917-1918). 


\section{Bibliographie sommaire}

Roland ALLENDER, 1914-1918 dans le Nord : surviure en pays occupé, SaintCyr-sur-Loire, A. Sutton, 2006, 126 p.

Pierre ARBEL, Mines. Exploitation des mines et Métallurgie, Paris, Impr. nationale, 1894, (97 p.) Exposition de Chicago en 1893. Rapports. Comité 12.]

Eric BUSSIÈRE, Patrice MARCILLOUX, Denis VARASCHIN (dir.), La Grande Reconstruction. Reconstruire le Pas-de-Calais après la Grande Guerre, Actes du colloque d'Arras, 8-10 novembre 2000, Arras, Archives départementales du Pas-de-Calais, 2002, 477 p.

Les Capitalistes en France: 1780-1914, présenté par Louis Bergeron, Paris, Gallimard, 1978, 233 p.

Dans les régions dévastées. L'CEurre de reconstitution et la solidarité française, Paris, Comité d'action des régions dévastées, 1925, 404 p.

Pierre DAUZET, Le Siècle des chemins de fer en France, 1821-1938, Fontenayaux-Roses, impr. de Bellenand, 1948, 379 p.

Jean FAVIER (dir.), Reconstructions et modernisation : la France après les ruines : 1918, 1945, exposition, Archives nationales, Hôtel de Rohan, janvier-mai 1991, Paris, Archives nationales, 1991, 310 p.

Odette HARDY-HEMERY, De la croissance à la désindustrialisation : un siècle dans le Valenciennois, Paris, Presses de la Fondation nationale des sciences politiques, 1984, $401 \mathrm{p}$.

Odette HARDY-HEMERY, Industries, patronat et ouvriers $d u$ Valenciennois pendant le premier $X X^{e}$ siècle : développements et restructurations capitalistes à l'age du charbon et de l'acier, Lille, Atelier national de reproduction des thèses de l'université Lille 3/Paris, Éditions Messidor, 1985, 5 vol., $4528 \mathrm{p}$.

Laure HENNEQUIN, «La Société Arbel de 1894 à 1939 : un demisiècle d'histoire technique, économique et sociale de la vie d'une entreprise française de construction mécanique », Villeneuve d'Ascq, maittrise d'histoire contemporaine, Lille 3, 1993, sous la direction de Mme HARDY-HEMERY, $382 \mathrm{p}$.

L'Industrie en France occupée, ouvrage établi par le grand quartier général allemand en 1916, Paris, Impr. nationale, 1923, 534 p.

Louis LE CHATELIER, Sowenirs 1873-1923, Paris, imprimerie Chaix, 1924, $194 \mathrm{p}$. 
A. LORBERT, La France an travail: le Nord, Paris, Pierre Roger, [1927], $294 \mathrm{p}$.

Alfred MORAIN, Le Bilan de trois ans de reconstitution, rapport présenté au Conseil général du Nord, Lille, impr. de L. Danel, 1922, 26 p.

Nos usines métallurgiques dévastées (1914-1918). Monographies de quelques grands usines métallurgiques françaises détruites par les Allemands, Paris, Éditions de la Revue de métallurgie, 1921, 234 p.

René ROMAN (dir.), Code des dommages de guerre. Lois et décrets. Circulaires et instructions ministérielles. Décisions de jurisprudence, Paris, Revue des dommages de guerre, Collection Roman, 1924, 259 p.

Michel ROUCHE (dir.), Histoire de Douai, Dunkerque, Éd. des Beffrois, $1985,347 \mathrm{p}$.

Gérard SIVERY (dir.), Histoire de Maubenge, Dunkerque, Éd. des Beffrois, 1984, $341 \mathrm{p}$.

\section{Périodiques :}

En Train (revue interne de la firme Alsthom).

L'Illustration.

La Passerelle (revue interne de la firme Arbel). 


\section{Annexe \\ Lettre des Établissements Arbel au président Wilson le 18 décembre 1918 ${ }^{71}$}

\section{AU PRÉSIDENT WILSON ARBITRE DE LA PAIX}

Monsieur le Président,

Les ÉTABLISSEMENTS ARBEL ont acquis successivement les terrains sur lesquels nous nous trouvons depuis 1893.

Leur superficie du début de 5 hectares s'est progressivement développée jusqu'à 55 hectares, et les 40 premiers ouvriers se sont développés jusqu'à atteindre le nombre de 2500 hommes, qui trouvaient ici, non seulement le pain quotidien, mais aussi l'aisance progressive, fruit d'un travail rémunérateur et d'une cordiale entente entre le Patron et ses ouvriers. J'en veux pour preuve les centaines de lettres qui, pendant ces quatre années de guerre me sont parvenues de tous les rangs de notre Personnel, déplorant les abominables dévastations dont nos usines étaient victimes, criant vengeance de toutes les infamies commises, et exprimant leur volonté de rentrer bientôt dans leur chère Usine, après la victoire complète et définitive contre une race de bandits.

Aujourd'hui, vous pouvez vous rendre compte, Monsieur le Président, que, de tant d'intelligence, de travail, de persévérance, de bonne volonté, il ne reste même pas des ruines, mais seulement de la poussière, du désert et du néant.

Tout a été, pendant quatre années, soigneusement déboulonné, méthodiquement emporté en Allemagne, d'abord les matières premières, puis les produits fabriqués, puis les machines, les engins de toute nature, la force motrice, les appareils électriques, les appareils de levage, le matériel roulant, enfin jusqu'aux bâtiments, aux charpentes, tout a été systématiquement enlevé, pillé, transporté, en dehors de toute raison de guerre.

71- AD Nord 10 R 4564. Dossier des dommages de guerre. Société Arbel. En réalité, ce voyage n'eut pas lieu et fut remplacé par une visite, en mars 1919, des journalistes attachés à la Conférence de la Paix. 
C'est systématiquement, froidement, dans le but avoué et proclamé de détruire une industrie française prospère et un concurrent dangereux pour l'exportation que les allemands ont, de sang froid, détruit notre industrie; je puis en fournir trois preuves absolues; la première réside dans le Rapport officiel de l'Ingénieur Schrodter, lu à l'Assemblée des Ingénieurs allemands de Dusseldorf, le 31 janvier 1915, dont ci-joint un exemplaire. Voici comment cet Ingénieur allemand appréciait notre Industrie :

«L'Usine qui s'est probablement le plus développée dans ces dernières années, est la fameuse Maison ARBEL, de DOUAI s'occupant presque uniquement de la fabrication de wagons spéciaux pour le transport en masses. C'est elle qui construit en grand nombre les déchargeurs automatiques de grande capacité pour le transport des minerais si connus de nos sidérurgistes du SudOuest. La carcasse de ces wagons est normalement formée de tôles embouties. Une aciérie nouvellement construite, avec deux fours MARTIN, des presses de forge des gros bloomings et à tôle produisent l'acier nécessaire. De grandes halles d'ateliers contenant cinq ou six presses hydrauliques et des machines-outils de toute forme et de toute grandeur, complètent l'installation, comprenant trois divisions séparées. Nos colonnes se sont installées dans les magasins, et maint atelier a dû servir d'écurie. Dans une cour, je voyais une importante colonne de boulangerie qui fabriquait de grandes quantités de pain bis pour l'armée. »

D’autre part, parmi les ingénieurs allemands qui sont venus rôder dans nos usines pour les dépecer, nous clouerons, au pilori de l'humanité M. KARLIER, associé à la Maison VAN DER ZIPPEN constructeur de wagons à KALK près COLOGNE; ce KARLIER et son beau-frère le capitaine allemand [vide] résidant à Valenciennes, et chargé de toute la destruction, s'adressant à notre ingénieur, M. FALCHI, et lui montrant notre grande presse de 1200 tonnes, 22 mètres de long, pièce unique au monde, lui dit textuellement ceci : "C'est avec cet engin que vous nous avez enlevé la commande de 100 wagons pétroliers roumains, nous allons l'emporter dans nos usines, et c'est nous qui ferons les wagons ARBEL, à votre place. »

Dès le lendemain, une équipe de prisonniers russes démontaient la dite presse et l'envoyaient en Allemagne.

Enfin, pendant trois mois, un Ingénieur Allemand a vérifié toutes nos archives, particulièrement les dessins de nos outillages, a mis de côté tout ce qu'il a pu juger lui être utile et qui a pris le chemin de l'Allemagne, le surplus a été odieusement brûlé, détruit, saccagé, et de cet énorme effort intellectuel, commercial et financier condensé dans 
nos archives, il ne reste plus que poussière ! C'est le fruit d'un labeur de 25 années, anéanti froidement, non dans le feu d'une bataille, mais dans le pillage calme et méthodique et continu d'une placide occupation.

Je crois donc la preuve surabondamment faite de la volonté formelle des allemands de détruire toute concurrence française. Ce qui a été vrai pour Douai l'est également pour tous les pays occupés.

Quelles en sont les conséquences.

Après quatre années de guerre, nous trouvons nos terrains dans l'état où nous les avons acheté il y a 25 ans ; les familles de nos 2500 ouvriers, de nos nombreux collaborateurs ont eu elles-mêmes tous leurs mobiliers volés, saccagés ou brûlés. Nous avons 33 millions de bons de réquisition donnés par les Allemands; une autre partie importante de notre fortune industrielle a été détruite sans aucune reconnaissance des bons de réquisition. En raison des prix actuels excessifs et de la rareté de toutes les matières, tout autant que la grandeur des besoins, il nous faudra sept à huit ans pour rétablir notre industrie. Ce ne sera plus 30 à 40 millions que nous aurons à dépenser pour cela comme la première fois en temps de paix, mais deux ou trois fois cette valeur. Pendant ce temps, l'Allemand qui, bien que vaincu aura maintenu et développé formidablement pendant la guerre ses instruments de travail, qui les aura accru de tous ceux qu'il nous aura volés poursuivra son action néfaste de développement dans le monde, en nous volant notre clientèle, comme il nous aura volé nos moyens d'action.

Pendant que le soldat allemand, redevenu ouvrier allemand se tiendra au chaud dans les mobiliers qu'il nous aura volés à nos classes ouvrières ou paysannes, celles-ci, après quatre années de misère sans nom, continueront à mourir de froid et de privations dans des maisons vidées, devant leurs usines détruites ou leurs champs dévastés.

Ceci est odieux, intolérable, inadmissible, plus odieux, plus intolérable que toutes les misères des combats. C'est pour cela, Monsieur le PRÉSIDENT, qu'il faut que tous les alliés imposent à l'Allemagne la reprise en nature, soit réelle, soit par compensation ou par similitude, et à défaut seulement, par compensation pécuniaire, afin de permettre plus rapidement à toutes les régions envahies des nations alliées leur résurrection économique.

Vous l'avez d'ailleurs promis, Monsieur le PRÉSIDENT, dans votre sublime proclamation faite à la Face du Monde Civilisé, et c'est pourquoi, en toute confiance, nous vous crions :

$$
\text { « JUSTICE ! JUSTICE !! JUSTICE !!! » }
$$


